\title{
URO-DYNAMIC EVALUATION
}

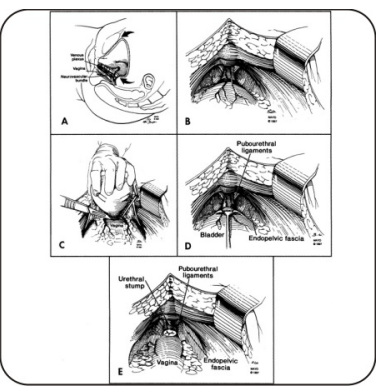

\section{DR. SAQUIB HANIF JANJUA, MBBS}

Registrar, Physical Medicine and Rehabilitation Armed Forces Institute of Rehabilitation Medicine Rawalpindi

DR. TARIQ MEHMOOD, MBBS

Resident, Physical Medicine and Rehabilitation Armed Forces Institute of Rehabilitation Medicine Rawalpindi

\section{DR. REHANA YASMIN, MBBS}

Registrar, Physical Medicine and Rehabilitation Armed Forces Institute of Rehabilitation Medicine Rawalpindi

\section{Dr. Zaheer Ahmad Gill, MBBS}

Registrar, Physical Medicine and Rehabilitation Armed Forces Institute of Rehabilitation Medicine Rawalpindi
Dr. M. Farooq Azam Rathore, MBBS

Resident, Physical Medicine and Rehabilitation Armed Forces Institute of Rehabilitation Medicine Rawalpindi.

\section{Dr. Maqsood UI Hasan MBBS, FCPS}

Consultant Physical Medicine and Rehabilitation, AFIRM, Rawalpindi

\section{Dr. Pervaiz Hasan Khan Niazi} MBBS, FICS, FCPS, D Med Rehab (UK)

Commandant and Senior Consultant, AFIRM, Rawalpindi

\section{Dr. Aamir Waheed Butt MBBS, FCPS}

Consultant Physical Medicine and Rehabilitation, AFIRM, Rawalpindi

\begin{abstract}
Objective: To describe the pattern of Urodynamic evaluation at Armed Forces Institute of Rehab Medicine, Rawalpindi. Study Design: Descriptive study. Materials and Methods: This study was conducted on 466 patients reporting for urodynamic evaluation at Urodynamic/Incontinence Clinic at A F Institute of Rehab Medicine from Feb 2003 to Dec 2006. We considered the age, gender, etiology, reason for referral, and presenting complaints whereas results were formulated according to Classification of voiding disorders by International society of Incontinence. Data was analyzed using statistical package for Social Science version 10. Results: In all (466) patients, $58 \%$ were males and $42 \%$ were females. Mean age was 46.8 years. Increased frequency (61\%) and incontinence (52\%) were the most common presenting complaints, $158(34 \%)$ had spinal cord injuries, 58 were cases of myelodysplasias and 128 reported with stress incontinence. Urodynamic studies showed that $152(33 \%)$ had hyperactive bladders, 110(24\%) had a contractile/hypo-contractile bladders, 104(22\%) had stress incontinence and
\end{abstract}


$56(12 \%)$ cases had normal results. Conclusion: Urodynamics helps in diagnosis of potentially life threatening urinary problems. The availability of this equipment and trained staff must be encouraged at tertiary care hospital. More research is required in this regard in Pakistan, which shall help in formulating better management protocols in future.

Key words: $\quad$ Urinary incontinence, Overactive bladder, Urodynamics

\section{INTRODUCTION}

Urodynamics is the study of transport, storage and evacuation of urine by the urinary tract ${ }^{1}$. The term Urodynamic was first used in 1953 by D.M Davis ${ }^{2}$ and since then the knowledge about urodynamic has progressed rapidly in two decades. The patients with urological complaints that are complex and which cannot be diagnosed with simple tests, suffer due to lack of urodynamics. For example a study by Hacker et al, showed that $40 \%$ of all $\mathrm{SCl}$ patients die of renal insufficiency if they were left completely untreated ${ }^{3}$. The urodynamics plays a key role in understanding, evaluation and management of voiding dysfunctions, especially when history clinical exam and simple tests are not sufficient for diagnosis and instillation of treatment.

The most common sequel of voiding disorders and the most frequent reason for referral is incontinence, which may be a result of bladder dysfunction, sphincter dysfunction, or a combination of both. The International Continence Society (ICS) defines incontinence as "Involuntary loss of urine is a social or hygienic problem and is objectively demonstrable ${ }^{4}$ ". There are various classification of voiding disorders but we prefer International Continence Society classification of voiding disorders because the storage and voiding phases are described separately 4 .

To the best of our knowledge in Pakistan, the urodynamics is currently available in few centers in Pakistan and there is lack of advanced urodynamic equipment and trained staff. Literature search on Medline \& local database 'Pakmedinet' with keywords 'urodynamic' and 'flowmetry' did not reveal even a single article pertaining to urodynamic evaluation in Pakistan. This study was carried out to describe the pattern of urodynamic evaluation at tertiary care rehabilitation centre and to highlight its role and importance in management of voiding disorders.

\section{MATERIAL AND METHODS}

The study was conducted at Urodynamic/Incontinence Clinic, Armed Forces Institute of Rehabilitation Medicine Rawalpindi, Pakistan (AFIRM).it was a 03-year descriptive case series. Patient inclusion was started in Feb 2003 to Dec 2006. 466 consecutive patients who reported for Urodynamic evaluation at AFIRM were selected after their consent had been taken. The study was approved by the hospital ethics committee at the start.

Patients of all ages and both genders were included. Cases with anorectal pathologies, supra pubic catheters in situ, altered behavior and no anticholinergic medications were excluded. The detailed history included a detailed personal profile, presenting complaints, time of onset of complaints, reason for referral, mode of bladder evacuation/voiding referring specialist, any previous surgery/trauma, gynecological problems (In females) and any neurological complaints. The clinical examination included a detailed neurological and urological examination. The investigations including, previous urodynamic studies (if available), ultrasound urinary bladder and kidneys, urine culture and sensitivity reports were noted on a specifically designed proforma for study purpose. The data was entered and saved in two separate computers.

The tests were performed on Dantec $₫$ Menuet 
Urodynamic Equipment. The various tests performed included Uroflowmetry for bladder outlet obstruction, water/voiding cystometries to rule out neurogenic bladders, abdominal leak point for patients who were unable to void and Valsalva Leak Point Pressures for stress incontinence. The uroflowmetry was performed on a spin disc flow-meter. The flow-metry was repeated twice to ascertain the results. Cystometries were performed by calculating intra vesical pressures by inserting two way, $8 \mathrm{Fr}$ and $10 \mathrm{Fr}$ disposal cystometry catheters and intra abdominal pressure by inserting disposable rectal balloon catheter. The EMG was recorded by catheter mounted ring electrode or surface EMG disposable catheter.

During cystometry, the fluid was infused at the rate of 30 $\mathrm{ml} / \mathrm{min}$ and was adjusted accordingly. The Uninhibited detrusor contraction was defined as the contraction resulting in rise of more than $15 \mathrm{~cm}$ of water. Hyperreflexia was a spontaneous or provoked, involuntary detrusor contractions leading to urinary incontinence during attempted suppression by the individual. Areflexia was defined when there was no definite detrusor concentration. Detrusor Sphincter Dyssynergia was defined when the detrusor contraction was accompanied with increased sphincteric EMG activity $^{1}$.

Leak point pressures 1 were checked in areflexic bladders. Those Bladder having leakage pressures of less than $40 \mathrm{cms}$ of water were labeled as low-pressure areflexic bladders where leakage occurred at more than $40 \mathrm{cms}$ of water or did not leak at all were labeled as high-pressure areflexic Bladders.

Valsalva Leak point pressures were measured to ascertain the type of stress incontinence. The bladder was filled to $250 \mathrm{ml}$ and patient was asked to generate increased abdominal pressures by performing Valsalva maneuver or coughing. If the leakage occurred at pressures, less than $60 \mathrm{~cm}$ of water the stress incontinence was labeled due to intrinsic sphincteric deficiency whereas leakage above $90 \mathrm{cms}$ of water was considered as due to hyper-mobile urethra. If leakage occurred between $60-90 \mathrm{Cm}$ of water, it was considered as mixed type of genuine stress incontinence.

\section{STATISTICAL ANALYSIS}

The data was recorded on proformas specifically designed for the study. The data was analyzed on SPSS version 10. The descriptive statistics were used to calculated mean and frequencies were calculated for string data.

\section{RESULTS}

\section{Gender and age}

A total of 466 new patients were evaluated by undergoing various Urodynamic tests. In all (466) patients 270(58\%) were males and 196(42\%) were females. Mean age was $46.8+11.16$ years (range 4-86 yrs). The peak of age distribution was seen in 50-60 yrs group (54\%). Two hundred and Ninety six patients were married, with no history of venereal or sexually transmitted diseases.

\section{Reason of Referral}

The most common reason for referral was incontinence followed by leakage of urine upon stress or during exertion. The various reasons for referral are shown in fig 1. Majority $56 \%$ was referred by urologists, $37 \%$ by Psychiatrists and $27 \%$ by gynecological. 402(86\%) had seven or more visits to general practitioners before they were referred for urodynamics. In all 466 patients, $278(59 \%)$ patients had restricted social activities due to Incontinence.

\section{Etiology}

In all 466 , there were $158(34 \%)$ cases of spinal cord injuries, $58(13 \%)$ cases of myelodysplasias, $128(27 \%)$ females presented with stress incontinence, 22(6\%) patients had stroke, 44(9\%) patients had signs and symptoms of Bladder outlet obstruction and $52(11 \%)$ cases presented other disorders. Of 152 patients exhibition detrusor hyperreflexia, 106(69\%) patients had 
Spinal cord injury, 24(15\%) had Myelodysplasia and $16(10 \%)$ cases had stroke. 06(3\%) cases had no diagnosed neurological disorders.

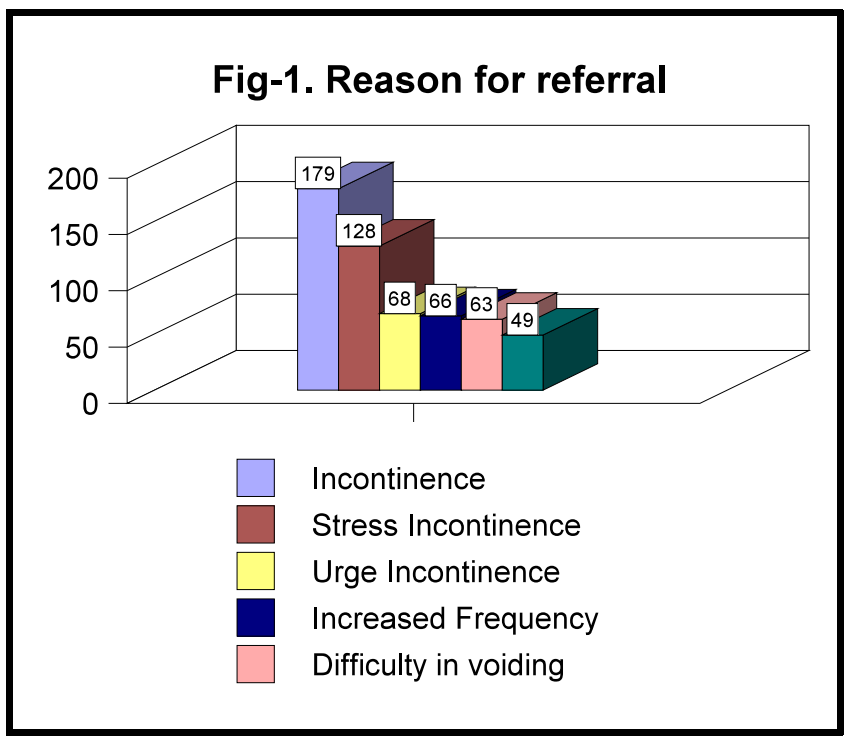

Of 110 cases of Hyporeflexic/Areflexic Bladders, $52(47 \%)$ had Spinal Cord injury, 34(31\%) had myelodysplasias, $6(5 \%)$ had stroke and $18(7 \%)$ had other neurological disorders including Gullian Barre Syndrome 6(5\%), Traumatic Brain Injury 6(5\%) and pelvic Trauma/Multiple injuries 2 (1.8\%). 4(3.5\%) had unexplained urinary retention in absence of any neurological disorder. The 128 females reporting for evaluation of stress incontinence included $65(50 \%)$ post menopausal women whereas $31(24 \%)$ experienced stress incontinence after childbirth. 22(17\%) had been operated upon pelvic/abdomen within last 02 years, whereas in 10(07\%) cases history was inadequate.

\section{Uroflowmetry}

This procedure was performed by asking the patient to void in spin disc Uroflowmeter at sensed urgency. A total of 44 uroflowmetries were performed and it was that 16(36\%) had severe Bladder Outlet obstruction, $9(20 \%)$ had moderate outlet obstruction, 15(34\%) had normal flow rates and in 4(9\%) patients uroflowmetry was invalid due to low volumes.

\section{Cystometries}

A total of 304 cystometries were performed and it was found that 152(33\%) had Hyperactive Bladders and $110(23 \%)$ had contractile/hypo-contractile Bladders. Among the Hyperactive Bladders 78(51\%) had detrusor Sphincter Dyssynergia (DSD) whereas 74(48\%) had uninhibited detrusor contractions only. Among 110 a contractile bladders, Abdominal leak point pressures showed that $79(71 \%)$ had high pressure bladders and $31(28 \%)$ had low intra-vesical pressures. (Table I)

\begin{tabular}{|l|c|c|c|}
\hline \multicolumn{4}{|c|}{ Table-I. Characteristics of Bladder } \\
\hline $\mathrm{n}=466$ & $\begin{array}{c}\text { Detrusor } \\
\text { hyperreflexia }\end{array}$ & $\begin{array}{c}\text { Areflexia/hy } \\
\text { poreflexia }\end{array}$ & $\begin{array}{c}\text { Elevated } \\
\text { ALPP } \mathbf{7 0} \\
\text { cms of } \\
\text { water }\end{array}$ \\
\hline Spinal Cord Injury & 106 & 52 & 112 \\
\hline Stroke & 16 & 6 & 7 \\
\hline Myelodysplaasias & 24 & 34 & 41 \\
\hline \multicolumn{4}{|c|}{ ALPP= Abdominal Leak Point Pressures } \\
\hline
\end{tabular}

\section{Valsalva Leak point Pressures: (AVLPP)}

AVLPP were performed in 128 patients presenting with stress incontinence. 39(30\%) had stress incontinence due to hyper-mobile urethra, $18(14 \%)$ due to intrinsic sphincter deficiency, 25(20\%)had mixed type of stress incontinence and 15(12\%) had stress induced urge incontinence. 31(24\%) exhibited no leakage at all and were declared normal. (Fig.2)

\section{Complications of the procedure}

In all $466(100 \%)$ patients, there were $03(.06 \%)$ cases of cervical Spinal cord Injury in which test was discontinued due to sings and symptoms of autonomic dyssreflexia. Only $12(2.5 \%)$ developed acute signs and symptoms of Urinary Tract Infection which was dealt with antibiotics and hydration. 
Fig-2. Results of valsalva leak point pressures

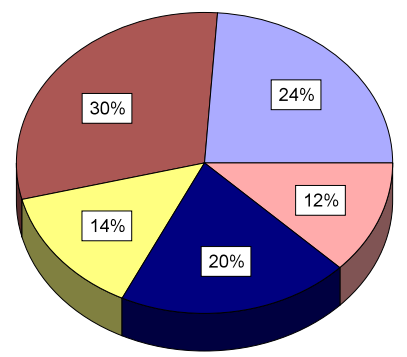

Normal

Hypermobile urethra

Intrinsic sphincter deficiency

Mixed type

Stress induced urge Incontinence

\section{DISCUSSION}

Urinary incontinence is cruel sequelae of various neurological and physiological disorders. It affects the patient's physical well being and has a negative role on patients self esteem by lowering the desire for social interactions ${ }^{5,6}$. Persistent urinary incontinence also increases the burden on care givers ${ }^{7}$ and predisposes the patients to medical complications ${ }^{8}$. In this study, $59 \%$ patients had restricted social activities due to Incontinence.

The exact cause and type of voiding disorder cannot be assessed by history and clinical examination alone, and it requires help of Urodynamics to ascertain the condition as a Storage disorder, voiding disorder or a combination of both. Various studies show that clinical examination alone was unable to provide definite guidelines for bladder management ${ }^{9,10}$. In our study $402(86 \%)$ had seven or more visits to general practitioners before they were referred for urodynamics. This finding was similar to developed countries as Wein and colleagues ${ }^{11}$ noted that in a survey of primary care physicians, $80 \%$ did not examine patients with urinary complaints; $30 \%$ of those patients received no assessment at all.
In our study Spinal cord injury was a major group and detrusor hyperreflexia was seen in $89 \%$ of patients with supra sacral injuries. When it was compared with other studies, detrusor hyperreflexia was the most common feature in supra sacral lesions and in stroke as well ${ }^{13}$.

The other interesting fact in our study was that $128(27 \%)$ females presented with stress incontinence. 31(24\%) patients had no evidence of stress incontinence whereas $30 \%$ females had stress incontinence due to hyper mobile urethra which is a treatable cause. Fifteen (12\%) patients had stress induces urge incontinence which was again a treatable cause. Among these 15 patients, seven had been operated for sling surgery for stress incontinence, but they had no relief.

The study reveals that there were $160(34 \%)$ high bladders (ALPP> $40 \mathrm{cms}$ of water) which if not treated may upper renal tract damage (Table-I). The procedure is very safe with low incidence of complications (3\%) despite of its invasive nature. The major limitation of this study was that no reference values for urodynamics have yet been formulated after research.

\section{CONCLUSION}

Urinary incontinence is not a major disability for patients but also for his care givers. Urodnamic evaluation can both diagnosis the underlying disorder and direct the accurate management. Urodynamic remains underdeveloped and underutilized in Pakistan. It is recommended that more research in this regard should be done to formulate reference values in our local population. We also recommend that urodynamics must be used as an essential tool, before taking a decision to operate upon the case of stress incontinence and neurogenic Bladders.

\section{REFERENCES}

1. Rodney A. Appell. Voiding Dysfunction. In: Victor W Nitti, Michael Ficazzola. Voiding Dysfunction: Diagnosis and Treatment. New Jersey: Humana Press Inc. 2000; pp. 3857 
2. Davis, D. M. The mechanisms of urologic disease. WB Saunders, Philadelphia1953.

3. Hackler RH. A 25-year prospective mortality study in the spinal cord injured patient: comparison with long term living paraplegic. J Uro1 1977; 117:486-488

4. Abrams P, Blaiva JG, Stanton SL, Anderson JT. The standardization of terminology of lower urinary tract function recommended by the International Continence Society. Intl urogynecol J 1990; 1:45

5. Bean JF, Kiely DK, Cairns KD, Morris JN. Influence of post stroke urinary incontinence on disability: the nursing home setting. Am J Phys Med Rehabil. 2003 Mar;82(3): 175-81.

6. Jawad $S H$, Ward $A B$ Jones $P$. Study of relationship between pre-morbid urinary incontinence and stroke functional outcomes. Clin rehabil 1999 Oct; 13(5): 44752.

7. Marinkovic S, Badlani G. Voiding and sexual dysfunction after cerebrovascular accidents. J Urol 2001 Feb; 165(2): 359-70.
8. Kowalczyk JJ. Office evaluation of the patient with an overactive Urinary bladder. J Am Osteopath Assoc 2000 Mar; 100(3 supp1): S1-4.

9. Weld KJ, Dmochowski RR. Association of level of injury and bladder behavior in patients with post-traumatic spinal cord injury. Urology. $2000 \mathrm{Apr}$; 55(4): 490-4.

10. Kaplan SA, Chancellor MB, Blaivas JG. Bladder and sphincter behavior in patients with spinal cord lesions. J Uro1. 1991 Jul; 146(1): 113-7.

11. Wein AJ, Rovner ES. The overactive bladder: an overview for primary care health providers. Int J Fertil 1999;44: 56-66.

12. Weld KJ, Dmochowski RR. Association of level of injury and bladder behavior in patients with post-traumatic spinal cord injury. Urology. $2000 \mathrm{Apr}$; 55(4): 490-4.

13. Tsuchida $\mathrm{S}$, Noto $\mathrm{H}$, yamaguchi $\mathrm{O}$, et al. Urodynamic studies on hemiplegic patients after cerebrovascular accident. Urology 1983 21:315-318.

\section{FOCUSED \& CONTINUOUS STRUGGLE LEADS TO SUCCESS \\ Shuja Tahir}

